International Journal of Pure and Applied Mathematics

Volume 109 No. 4 2016, 847-860

ISSN: 1311-8080 (printed version); ISSN: 1314-3395 (on-line version)

url: http://www.ijpam.eu

doi: 10.12732/ijpam.v109i4.8

\title{
SPECTRAL THEORY FOR INTEGRATED SEMIGROUPS
}

\author{
A. Tajmouati ${ }^{\S}, \mathrm{H}$. Boua ${ }^{2}$ \\ ${ }^{1,2}$ Faculty of Sciences \\ Sidi Mohamed Ben Abdellah Univeristy \\ Dhar Al Mahraz Fez, MOROCCO
}

\begin{abstract}
In this paper, we investigate the transfer of some spectral properties from the integrated semigroup to its generator.
\end{abstract}

AMS Subject Classification: 47B47, 47B20, 47B10

Key Words: integrated semigroup, descend, ascent, Drazin spectrum, Kato spectrum, essential Kato spectrum

\section{Introduction and Preliminaries}

Throughout this work, $X$ denotes a complex Banach space and $\mathcal{B}(X)$ denotes the Banach algebra of all bounded linear operators on $X$. Let $T$ a closed operator with domain $D(T)$, we denote by $T^{*}, R(T), N(T), R^{\infty}(T)=\bigcap_{n \geq 0} R\left(T^{n}\right)$, $\rho(T), \sigma(T), \sigma_{p}(T), \sigma_{r}(T), \sigma_{k}(T), \sigma_{e s}(T)$ respectively the adjoint, the range, the kernel, the hyper-range, the resolvent set, the spectrum, the point spectrum, the residual spectrum, the Kato spectrum and the essential kato spectrum of $T$.

$\begin{array}{lr}\text { Received: } & \text { May 10, 2016 } \\ \text { Revised: } & \text { September 28, 2016 } \\ \text { Published: } & \text { October 7, 2016 }\end{array}$

(c) 2016 Academic Publications, Ltd. url: www.acadpubl.eu

${ }^{\S}$ Correspondence author 
The ascent of $T$ is defined by $a(T)=\min \left\{p \in \mathbb{N}: N\left(T^{p}\right)=N\left(T^{p+1}\right)\right\}$; if such $p$ does not exist, we let $a(T)=\infty$. Similarly, the descent of $T$ is $d(T)=\min \left\{q \in \mathbb{N}: R\left(T^{q}\right)=R\left(T^{q+1}\right)\right\}$, if such $q$ does not exist we let $d(T)=\infty$ [3]. It is well known that if both $a(T)$ and $d(T)$ are finite then $a(T)=d(T)$ and therefore we have the decomposition $X=R\left(T^{p}\right) \oplus N\left(T^{p}\right)$ where $p=a(T)=$ $d(T)$.

The descend and ascent spectrum defined by:

$$
\begin{gathered}
\sigma_{\text {desc }}(T)=\{\lambda \in \mathbb{C}: d(\lambda-T)=\infty\} \\
\sigma_{\text {asc }}(T)=\{\lambda \in \mathbb{C}: a(\lambda-T)=\infty\}
\end{gathered}
$$

The essential ascent and descend of $T$ are defined respectively by:

$$
\begin{gathered}
d_{e}(T)=\min \left\{n \in \mathbb{N}: \operatorname{dim} R\left(T^{n}\right) / R\left(T^{n+1}\right)<\infty\right\} \\
a_{e}(T)=\min \left\{n \in \mathbb{N}: \operatorname{dim} N\left(T^{n+1}\right) / N\left(T^{n}\right)<\infty\right\}
\end{gathered}
$$

The essential ascent and descend spectrum are defined respectively by:

$$
\begin{aligned}
& \sigma_{\text {asc }}^{e}(T)=\left\{\lambda \in \mathbb{C}: a_{e}(\lambda-T)=\infty\right\} \\
& \sigma_{\text {des }}^{e}(T)=\left\{\lambda \in \mathbb{C}: d_{e}(\lambda-T)=\infty\right\}
\end{aligned}
$$

Recall that $T$ is a Drazin invertible if $d(T)<\infty$ and $a(T)<\infty$. The Drazin spectrum is $\sigma_{D}(T)=\{\lambda \in \mathbb{C}: d(\lambda-T)=\infty$ or $a(\lambda-T)=\infty\}$.

The set of all upper semi-Fredholm operators is defined by:

$$
\Phi_{+}(X)=\{T \in B(X): \operatorname{dim} N(\lambda-T)<\infty \text { and } R(\lambda-T) \text { is closed }\}
$$

The upper semi-Fredholm spectrum defined by:

$$
\sigma_{\Phi_{+}}(T)=\left\{\lambda \in \mathbb{C}: \lambda-T \notin \Phi_{+}(X)\right\}
$$

while the set of all lower semi-Fredholm operators is defined by:

$$
\Phi_{-}(X)=\{T \in B(X): \operatorname{codim} R(\lambda-T)<\infty\}
$$

The lower semi-Fredholm spectrum defined by:

$$
\sigma_{\Phi_{-}}(T)=\left\{\lambda \in \mathbb{C}: \lambda-T \notin \Phi_{-}(X)\right\}
$$

The set of all semi-Fredholm operators is defined by:

$$
\Phi_{ \pm}(X)=\Phi_{+}(X) \cup \Phi_{-}(X)
$$


The semi-Fredholm spectrum defined by:

$$
\sigma_{\Phi_{ \pm}}(T)=\left\{\lambda \in \mathbb{C}: \lambda-T \notin \Phi_{ \pm}(X)\right\}
$$

The class $\Phi(X)$ of all Fredholm operators is defined by:

$$
\Phi(X)=\Phi_{+}(X) \cap \Phi_{-}(X)
$$

The semi-Fredholm spectrum defined by:

$$
\sigma_{\Phi}(T)=\sigma_{\Phi_{+}}(T) \cup \sigma_{\Phi-}(T)
$$

The class of all upper semi-Browder operators on a Banach space $X$ that is defined by:

$$
\mathcal{B}_{+}(X)=\left\{T \in \Phi_{+}(X): a(T)<\infty\right\}
$$

The upper semi-Browder spectrum defined by:

$$
\sigma_{\mathcal{B}_{+}}(T)=\left\{\lambda \in \mathbb{C}: \lambda-T \notin \mathcal{B}_{+}(X)\right\}
$$

The class of all lower semi-Browder operators that is defined by:

$$
\mathcal{B}_{-}(X)=\left\{T \in \Phi_{-}(X): d(T)<\infty\right\}
$$

The lower semi-Browder spectrum defined by:

$$
\sigma_{\mathcal{B}_{-}}(T)=\left\{\lambda \in \mathbb{C}: \lambda-T \notin \mathcal{B}_{-}(X)\right\}
$$

The class of all Browder operators is defined by:

$$
\mathcal{B}(X)=\mathcal{B}_{+}(X) \cap \mathcal{B}_{-}(X)=\{T \in \Phi(X): a(T)<\infty \text { and } d(T)<\infty\}
$$

The Browder spectrum defined by:

$$
\sigma_{\mathcal{B}}(T)=\{\lambda \in \mathbb{C}: \lambda-T \notin \mathcal{B}(X)\}
$$

Recall that $T$ is said to be Kato operator or semi-regular if $R(T)$ is closed and $N(T) \subseteq R^{\infty}(T)$. The set $\rho_{\gamma}(T)=\{\lambda \in \mathbb{C}: T-\lambda I$ is Kato $\}$ denotes the Kato resolvent and $\sigma_{\gamma}(T)=\mathbb{C} \backslash \rho_{\gamma}(T)$ the Kato spectrum of $T$. It is well known that $\rho_{\gamma}(T)$ is an open subset of $\mathbb{C}$.

$T$ is essential Kato if $R(T)$ is closed and there exists a subspace $L$ in $X$ with $\operatorname{dim} L<\infty$ such that $N(T) \subseteq R^{\infty}(T)+L$. The essential Kato spectrum of $T$ is defined by $\sigma_{\gamma}^{e}(T)=\{\lambda \in \mathbb{C}: \lambda-T$ is not a essential Kato operator $\}$. 
Integrated semigroups were first defined by Arendt [1] for integer-valued $\alpha$. Arendt showed that certain natural classes of operators, such as adjoint semigroups of $C_{0}$ semigroups on non-reflexive Banach spaces, give rise to integrated semigroups which are not integrals of $C_{0}$ semigroups.

A family of bounded linear operators $(S(t))_{t \geq 0}$, on a Banach space $\mathrm{X}$ is called an integrated semigroup iff:

i) $S(0)=0$.

ii) $S(t)$ is strongly continuous in $t \geq 0$.

iii) $S(r) S(t)=\int_{0}^{r}(S(\tau+t)-S(\tau)) d \tau=S(t) S(r)$.

The differentation spaces $C^{n}, n \geq 0$, are defined by $C^{0}=X$ and

$$
C^{n}=\left\{x \in X: S(.) x \in C^{n}\left(\mathbb{R}^{+} ; X\right)\right\}
$$

Using this notion iii) can equivalently be formulated by $S(t) x \in C^{1}$ and $S^{\prime}(r) S(t)$ $=S(r+t)-S(r)$.

The set $N=\{x \in X ; S(t) x=0, \forall t \geq 0\}$ is called the degeneration space of the integrated semigroup $(S(t))_{t \geq 0} . \quad(S(t))_{t \geq 0}$ is called non-degenerate if $N=\{0\}$ and degenerate otherwise.

The generator $A: D(A) \subseteq X \rightarrow X$ of a non-degenerate integrated semigroup $(S(t))_{t \geq 0}$ is defined as follows: $x \in D(A)$ and $A x=y$ iff $x \in C^{1}$ and $S^{\prime}(t) x-x=S(t) y$ for $t \geq 0$.

$C^{2} \subseteq D(A) \subseteq C^{1}$ and $A x=S^{\prime \prime}(0) x$ for $X \in C^{2}$. Moreover $A C^{2} \subseteq C^{1}$.

$A$ is a closed linear operator. $S(t): C^{1} \rightarrow C^{2} \subseteq D(A)$ and $A S(t) x=$ $S^{\prime \prime}(0) S(t) x=S^{\prime}(t) x-x$.

Further $A S(t) x=S(t) A x$ for $x \in D(A)$.

$\int_{0}^{t} S(r) d r$ maps $X$ into $D(A)$ and $A \int_{0}^{t} S(r) x d r=S(t) x-t x$.

A non-degenerate integrated semigroup is uniquely determined by its generator.

Let $u:[0, T) \rightarrow X$ be continuous such that

$$
\int_{0}^{t} u(s) d s \in D(A) \text { and } A\left(\int_{0}^{t} u(s) d s\right)=u(t),
$$

for $0 \leq t \leq T$. Then $u=0$ in $[0, T)$.

Arendt [1] showed that if $A$ generates $S_{t}$ as an $n$-times integrated semigroup, then the Abstract Cauchy Problem $u^{\prime}(t)=A u(t), u(0)=x$ has a classical solution for all $x \in D\left(A^{n+1}\right)$. 
In the theory of strongly continuous semigroups, some authors motivated by the relationships between the strongly continuou semigroup and its generator, like the spectral inclusion $[5,2]$. In the next section, we investigate the transfer of some spectral properties from the integrated semigroup to its generator. After, we will prove the spectral inclusion for some spectra.

\section{Main Results}

In the sequel, we will prove that the following lemma holds.

Lemma 2.1. Let $A$ be the generator of an integrated semigroup $(S(t))_{t \geq 0}$. Then, for all $\lambda \in \mathbb{C}, t \geq 0$, and $n \in \mathbb{N}$,

1. $\left(\int_{0}^{t} e^{\lambda s} d s-S(t)\right) x=(\lambda-A) D_{\lambda}(t) x, \forall x \in X$.

2. $\left(\int_{0}^{t} e^{\lambda s} d s-S(t)\right) x=D_{\lambda}(t)(\lambda-A) x, \forall x \in D(A)$.

3. $R\left(\int_{0}^{t} e^{\lambda s} d s-S(t)\right)^{n} \subseteq R(\lambda-A)^{n}$.

4. $N\left((\lambda-A)^{n}\right) \subseteq N\left(\int_{0}^{t} e^{\lambda s} d s-S(t)\right)^{n}$.

Proof. 1. For all $r, t \in[0,+\infty[$ and $x \in X$ we have

$$
\begin{aligned}
S(r) D_{\lambda}(t) x & =S(r) \int_{0}^{t} e^{\lambda(t-s)} S(s) x d s \\
& =\int_{0}^{t} e^{\lambda(t-s)} S(r) S(s) x d s \\
& =\int_{0}^{t} \int_{0}^{r} e^{\lambda(t-s)}[S(\tau+s)-S(\tau)] x d \tau d s \\
& =\int_{0}^{r} \int_{0}^{t} e^{\lambda(t-s)}[S(\tau+s)-S(\tau)] x d s d \tau
\end{aligned}
$$

Then, for all $x \in X, D_{\lambda}(t) x \in C^{1}$ and

$$
\begin{aligned}
\frac{d}{d r} S(r) D_{\lambda}(t) x= & \int_{0}^{t} e^{\lambda(t-s)}[S(r+s)-S(r)] x d s \\
= & \int_{0}^{t} e^{\lambda(t-s)}[S(r+s)-S(s)] x d s+\int_{0}^{t} e^{\lambda(t-s)} S(s) x d s \\
& -\int_{0}^{t} e^{\lambda(t-s)} S(r) x d s
\end{aligned}
$$




$$
\begin{aligned}
& =\int_{0}^{t} e^{\lambda(t-s)} \frac{d}{d s}[S(s) S(r)] x d s-S(r) \int_{0}^{t} e^{\lambda s} x d s+D_{\lambda}(t) x \\
& =S(r) S(t) x+\lambda S(r) D_{\lambda}(t) x-S(r) \int_{0}^{t} e^{\lambda s} x d s+D_{\lambda}(t) x \\
& =S(r)\left[S(t)+\lambda D_{\lambda}(t) x-\int_{0}^{t} e^{\lambda s} x d s\right]+D_{\lambda}(t) x
\end{aligned}
$$

Therefore $D_{\lambda}(t) x \in D(A)$ and $A D_{\lambda}(t) x=S(t)+\lambda D_{\lambda}(t) x-\int_{0}^{t} e^{\lambda s} x d s$.

Thus

$$
\left(\int_{0}^{t} e^{\lambda s} d s-S(t)\right) x=(\lambda-A) D_{\lambda}(t) x .
$$

2. For all $x \in D(A)$, we have

$$
\begin{aligned}
D_{\lambda}(t) A x & =\int_{0}^{t} e^{\lambda(t-s)} S(s) A x d s \\
& =\int_{0}^{t} e^{\lambda(t-s)}\left(S^{\prime}(s) x-x\right) d s \\
& =\int_{0}^{t} e^{\lambda(t-s)} S^{\prime}(s) x d s-\int_{0}^{t} e^{\lambda s} x d s \\
& =S(s)+\lambda D_{\lambda}(t) x-\int_{0}^{t} e^{\lambda s} x d s
\end{aligned}
$$

Hence

$$
\left(\int_{0}^{t} e^{\lambda s} d s-S(t)\right) x=D_{\lambda}(t)(\lambda-A) x .
$$

Theorem 1. Let $(S(t))_{t \geq 0}$ be an integrated semigroup and $A$ be its generator. Then

$$
\int_{0}^{t} e^{s \sigma(A)} d s \subseteq \sigma(S(t)), \int_{0}^{t} e^{s \sigma_{p}(A)} d s \subseteq \sigma_{p}(S(t))
$$

Proof. 1. Let $\int_{0}^{t} e^{s \lambda} d s \in \rho(S(t))$ and let $L=\left(\int_{0}^{t} e^{\lambda s} d s-S(t)\right)^{-1}$. The operator $D_{\lambda}(t)$ and $L$ commute. From lemma2.1, we deduce

$$
(\lambda-A) D_{\lambda}(t) L x=x \text { for every } x \in X
$$

and

$$
L(\lambda-A) D_{\lambda}(t) x=x \text { for every } x \in D(A)
$$


Since $D_{\lambda}(t)$ and $L$ commute we also have

$$
(\lambda-A) L D_{\lambda}(t) x=x \text { for every } x \in D(A)
$$

Therefore, $\lambda \in \rho(A)$ and $\int_{0}^{t} e^{s \sigma(A)} d s \subseteq \sigma(S(t))$

2. If $\lambda \in \sigma_{p}(A)$ then there is an $x_{0} \in D(A), x_{0} \neq 0$, such that $(A-\lambda I) x_{0}=0$. From lemma2.1 it then follows that $\left(\int_{0}^{t} e^{\lambda s} d s-S(t)\right) x_{0}=0$ and therefore $\int_{0}^{t} e^{s \lambda} d s \in \sigma_{p}(S(t))$.

Lemma 2.2. Let $(S(t))_{t \geq 0}$ an integrated semigroup on $X$ with generator $A$. For $\lambda \in \mathbb{C}$ and $t \geq 0$, let $L_{\lambda}(t) x=\int_{0}^{t} e^{-\lambda s} D_{\lambda}(s) x d s$, then

1. $L_{\lambda}(t)$ is a bounded linear operator on $X$.

2. $\forall x \in X, L_{\lambda}(t) x \in D(A)$ and $(\lambda-A) L_{\lambda}(t)+G_{\lambda}(t) D_{\lambda}(t)=\phi_{\lambda}(t) I$ with $G_{\lambda}(t)=e^{-\lambda t} I$ and $\phi_{\lambda}(t)=\int_{0}^{t} \int_{0}^{\tau} e^{-\lambda \sigma} x d \sigma d \tau$.

3. The operators $L_{\lambda}(t), G_{\lambda}(t), D_{\lambda}(t)$ and $(\lambda-A)$ are pairwise commute.

Proof. 1. Obvious.

2. For all $r \geq 0$ we have:

$$
\begin{aligned}
S(r) L_{\lambda}(t) x & =\int_{0}^{t} e^{-\lambda \tau} S(r) D_{\lambda}(\tau) x d \tau \\
& =\int_{0}^{t} \int_{0}^{\tau} e^{-\lambda \sigma} \int_{0}^{r}[S(u+\sigma)-S(u)] x d u d \sigma d \tau \\
& =\int_{0}^{t} \int_{0}^{\tau} \int_{0}^{r} e^{-\lambda \sigma}[S(u+\sigma)-S(u)] x d u d \sigma d \tau \\
& =\int_{0}^{r} \int_{0}^{t} \int_{0}^{\tau} e^{-\lambda \sigma}[S(u+\sigma)-S(u)] x d \sigma d \tau d u
\end{aligned}
$$

Therefore, for all $x \in X, L_{\lambda}(t) x \in C^{1}$ and

$$
\begin{aligned}
\frac{d}{d r} S(r) L_{\lambda}(t) x= & \int_{0}^{t} \int_{0}^{\tau} e^{-\lambda \sigma}[S(r+\sigma)-S(r)] x d \sigma d \tau \\
= & \int_{0}^{t} \int_{0}^{\tau} e^{-\lambda \sigma}[S(r+\sigma)-S(\sigma)] x d \sigma d \tau+L_{\lambda}(t) x \\
& -S(r) \phi_{\lambda}(t)
\end{aligned}
$$




$$
\begin{aligned}
& =\int_{0}^{t} \int_{0}^{\tau} e^{-\lambda \sigma} \frac{d}{d \sigma} S(\sigma) S(r) x d \sigma d \tau+L_{\lambda}(t) x-S(r) \phi_{\lambda}(t) \\
& =S(r)\left[e^{-\lambda t} D_{\lambda}(t) x+\lambda L_{\lambda}(t) x-\phi_{\lambda}(t) x\right]+L_{\lambda}(t) x
\end{aligned}
$$

Therefore, $A L_{\lambda}(t) x=e^{-\lambda t} D_{\lambda}(t) x+\lambda L_{\lambda}(t) x-\phi_{\lambda}(t) x$.

$(\lambda-A) L_{\lambda}(t)+G_{\lambda}(t) D_{\lambda}(t)=\phi_{\lambda}(t) I$ with $G_{\lambda}(t)=e^{-\lambda t} I$.

3. For all $t \geq 0, L_{\lambda}(t)$ and $D_{\lambda}(t)$ commuting.

Indeed, for $t, s \geq 0$ we have:

$$
\begin{aligned}
D_{\lambda}(t) D_{\lambda}(s) x & =\int_{0}^{t} e^{\lambda(t-u)} S(u) D_{\lambda}(s) x d u \\
& =\int_{0}^{t} e^{\lambda(t-u)} S(u) \int_{0}^{s} e^{\lambda(s-v)} S(v) x d v d u \\
& =\int_{0}^{t} \int_{0}^{s} e^{\lambda(t-u)} e^{\lambda(s-v)} S(u) S(v) x d v d u \\
& =\int_{0}^{s} e^{\lambda(s-v)} S(v) \int_{0}^{t} e^{\lambda(t-u)} S(u) x d u d v \\
& =D_{\lambda}(s) D_{\lambda}(t) x
\end{aligned}
$$

Therefore:

$$
\begin{aligned}
L_{\lambda}(t) D_{\lambda}(t) x & =\int_{0}^{t} e^{-\lambda u} D_{\lambda}(u) D_{\lambda}(t) x d u \\
& =\int_{0}^{t} e^{-\lambda u} D_{\lambda}(t) D_{\lambda}(u) x d u \\
& =D_{\lambda}(t) \int_{0}^{t} e^{-\lambda u} D_{\lambda}(u) x d u \\
& =D_{\lambda}(t) L_{\lambda}(t) x
\end{aligned}
$$

For all $x \in D(A)$ we have

$$
\begin{aligned}
L_{\lambda}(t)(\lambda-A) x & =\int_{0}^{t} e^{-\lambda s} D_{\lambda}(s)(\lambda-A) x d s \\
& =\int_{0}^{t} e^{-\lambda s}\left(e^{\lambda s}-S(s)\right) x d s \\
& =\phi_{\lambda}(t) x-\int_{0}^{t} e^{-\lambda s} S(s) x d s \\
& =\phi_{\lambda}(t) x-G_{\lambda}(t) D_{\lambda}(t) x
\end{aligned}
$$




$$
=(\lambda-A) L_{\lambda}(t) x
$$

For all $x \in D(A)(\lambda-A) G_{\lambda}(t) x=G_{\lambda}(t)(\lambda-A) x$ obvious.

For all $x \in D(A)(\lambda-A) D_{\lambda}(t) x=D_{\lambda}(t)(\lambda-A) x$ see lemma 2.1

Lemma 2.3. Let $(S(t))_{t \geq 0}$ an integrated semigroup on $X$ with generator $A$. If $R\left(\int_{0}^{t} e^{\lambda s} d s-S(t)\right)$ is a closed, then $R(\lambda-A)$ is closed.

Proof. Let $y_{n}=(\lambda-A) x_{n}$ be a convergent sequence with limit $y \in X$, according to (1) we have $\phi_{\lambda}(t) x_{n}=(\lambda-A) L_{\lambda}(t) x_{n}+G_{\lambda}(t) D_{\lambda}(t) x_{n}$ and $\phi_{\lambda}(t) y_{n}=$ $(\lambda-A) L_{\lambda}(t) y_{n}+G_{\lambda}(t)\left(\int_{0}^{t} e^{\lambda s} d s-S(t)\right) x_{n}$. Then:

$\left(\int_{0}^{t} e^{\lambda s} d s-S(t)\right) G_{\lambda}(t) x_{n}=y_{n}-(\lambda-A) L_{\lambda}(t) y_{n}$ tends to $\phi_{\lambda}(t) y-(\lambda-$ A) $L_{\lambda}(t) y \in R\left(\int_{0}^{t} e^{\lambda s} d s-S(t)\right)$ since $(\lambda-A) L_{\lambda}(t)$ is a linear bounded operator and $R\left(\int_{0}^{t} e^{\lambda s} d s-S(t)\right)$ is closed. Then there exists $z \in X$ such that $\phi_{\lambda}(t) y-$ $(\lambda-A) L_{\lambda}(t) y=\left(\int_{0}^{t} e^{\lambda s} d s-S(t)\right) z$ then $y=(\lambda-A)\left[L_{\lambda}(t) y+D_{\lambda}(t) z\right]$, hence $y \in R(\lambda-A)$.

Theorem 2. Let $(S(t))_{t \geq 0}$ an integrated semigroup on $X$ with generator $A$. Then for all $t>0$ we have

1. If $\int_{0}^{t} e^{\lambda s} d s-S(t)$ is a Kato operator, then $\lambda-A$ is a Kato operator.

2. If $\int_{0}^{t} e^{\lambda s} d s-S(t)$ is a essential Kato operator, then $\lambda-A$ is a essential Kato operator.

Proof. 1. Suppose that $\int_{0}^{t} e^{\lambda s} d s-S(t)$ is a Kato operator, then for all $n \in$ $\mathbb{N}$, we have $N\left(\int_{0}^{t} e^{\lambda s} d s-S(t)\right) \subseteq R\left(\int_{0}^{t} e^{\lambda s} d s-S(t)\right)^{n}$ and $R\left(\int_{0}^{t} e^{\lambda s} d s-S(t)\right)$ is closed. According to lemma2.1 and lemma2.3 we have $N(\lambda-A) \subseteq$ $N\left(\int_{0}^{t} e^{\lambda s} d s-S(t)\right) \subseteq R\left(\int_{0}^{t} e^{\lambda s} d s-S(t)\right)^{n} \subseteq R(\lambda-A)^{n}$ and $R(\lambda-A)$ is closed.

2. Suppose that $\int_{0}^{t} e^{\lambda s} d s-S(t)$ is a essential Kato operator, then there exists a subspace $L$ in $X$ such that $\operatorname{dim} L<\infty, N\left(\int_{0}^{t} e^{\lambda s} d s-S(t)\right) \subseteq$ $R^{\infty}\left(\int_{0}^{t} e^{\lambda s} d s-S(t)\right)+L$ and $R\left(\int_{0}^{t} e^{\lambda s} d s-S(t)\right)$ is closed. According to lemma2.1 and lemma2.3 we have $N(\lambda-A) \subseteq N\left(\int_{0}^{t} e^{\lambda s} d s-S(t)\right) \subseteq$ $R^{\infty}\left(\int_{0}^{t} e^{\lambda s} d s-S(t)\right)+L \subseteq R^{\infty}(\lambda-A)+L$ and $R(\lambda-A)$ is closed, hence $\lambda-A$ is essential Kato operator 
Corollary 1. Let $S(t)_{t \geq 0}$ be an integrated semigroup on $X$ with generator A. Then for all $t>0$ :

$$
\int_{0}^{t} e^{s \sigma_{\gamma}(A)} d s \subseteq \sigma_{\gamma}(S(t)) \text { and } \int_{0}^{t} e^{s \sigma_{\gamma}^{e}(A)} d s \subseteq \sigma_{\gamma}^{e}(S(t))
$$

Theorem 3. Let $(S(t))_{t \geq 0}$ an integrated semigroup on $X$ with generator A. Then for all $t>0$ we have

1. If $\int_{0}^{t} e^{\lambda s} d s-S(t)$ is a upper semi-Fredholm operator, then $\lambda-A$ is a upper semi-Fredholm operator.

2. If $\int_{0}^{t} e^{\lambda s} d s-S(t)$ is a lower semi-Fredholm operator, then $\lambda-A$ is a lower semi-Fredholm operator.

3. If $\int_{0}^{t} e^{\lambda s} d s-S(t)$ is a semi-Fredholm operator, then $\lambda-A$ is a semiFredholm operator.

4. If $\int_{0}^{t} e^{\lambda s} d s-S(t)$ is a Fredholm operator, then $\lambda-A$ is a Fredholm operator.

Proof. 1. If $\int_{0}^{t} e^{\lambda s} d s-S(t)$ is a upper semi-Fredholm operator, then $\operatorname{dim} N\left(\int_{0}^{t} e^{\lambda s} d s-S(t)\right)<\infty$ and $R\left(\int_{0}^{t} e^{\lambda s} d s-S(t)\right)$ is closed. According to lemma2.1 and lemma 2.3 we have $\operatorname{dim}(\lambda-A)<\infty$ and $R(\lambda-A)$ is closed, hence $\lambda-A$ is a upper semi-Fredholm operator.

2. If $\int_{0}^{t} e^{\lambda s} d s-S(t)$ is a lower semi-Fredholm operator, then $\operatorname{codim} R\left(\int_{0}^{t} e^{\lambda s} d s-\right.$ $S(t))<\infty$. According to lemma2.1 we have codim $R(\lambda-A)<\infty$, hence $\lambda-A$ is a lower semi-Fredholm operator.

3. Obvious.

4. Obvious.

Corollary 2. Let $S(t)_{t \geq 0}$ be an integrated semigroup on $X$ with generator A. Then for all $t>0$ :

$$
\begin{gathered}
\int_{0}^{t} e^{s \sigma_{\Phi_{+}}(A)} d s \subseteq \sigma_{\Phi_{+}}(S(t)), \int_{0}^{t} e^{s \sigma_{\Phi_{-}}(A)} d s \subseteq \sigma_{\Phi_{-}}(S(t)) \\
\int_{0}^{t} e^{s \sigma_{\Phi_{ \pm}}(A)} d s \subseteq \sigma_{\Phi_{ \pm}}(S(t)), \int_{0}^{t} e^{s \sigma_{\Phi}(A)} d s \subseteq \sigma_{\Phi}(S(t))
\end{gathered}
$$

Theorem 4. Let $(S(t))_{t \geq 0}$ an integrated semigroup on $X$ with generator $A$. Then for all $t>0$ we have

1. If $d\left(\int_{0}^{t} e^{\lambda s} d s-S(t)\right)<\infty$, then $d(\lambda-A)<\infty$. 
2. If $a\left(\int_{0}^{t} e^{\lambda s} d s-S(t)\right)<\infty$, then $a(\lambda-A)<\infty$.

3. If $d_{e}\left(\int_{0}^{t} e^{\lambda s} d s-S(t)\right)<\infty$, then $d_{e}(\lambda-A)<\infty$.

4. If $a_{e}\left(\int_{0}^{t} e^{\lambda s} d s-S(t)\right)<\infty$, then $a_{e}(\lambda-A)<\infty$.

5. If $\int_{0}^{t} e^{\lambda s} d s-S(t)$ is a Drazin invertible, then $\lambda-A$ is a Drazin invertible.

Proof. 1. If $d\left(\int_{0}^{t} e^{\lambda s} d s-S(t)\right)<\infty$, then there exists $n \in \mathbb{N}$ such that

$$
R\left(\int_{0}^{t} e^{\lambda s} d s-S(t)\right)^{n}=R\left(\int_{0}^{t} e^{\lambda s} d s-S(t)\right)^{n+1}
$$

There exists two operators $L_{n}(t)$ and $G_{n}(t)$ such that:

$$
(\lambda-A)^{n} L_{n}(t)+G_{n}(t) B_{\lambda}^{n}(t)=I
$$

and $L_{n}(t), H_{n}(t), D_{\lambda}(t)$ and $(\lambda-A)$ are pairwise commute.

Let $y \in R(\lambda-A)^{n}$ and $x \in D\left(A^{n}\right)$ such that $y=(\lambda-A)^{n} x$. According to (1) we have:

$$
\begin{aligned}
(\lambda-A)^{n} x & =(\lambda-A)^{n} L_{n}(t)(\lambda-A)^{n} x+G_{n}(t) D_{\lambda}^{n}(t)(\lambda-A)^{n} x \\
& =(\lambda-A)^{n+1} L_{n}(t)(\lambda-A)^{n-1} x+G_{n}(t)\left(\int_{0}^{t} e^{\lambda s} d s-S(t)\right)^{n} x
\end{aligned}
$$

Let $z \in X$ such that $\left(\int_{0}^{t} e^{\lambda s} d s-S(t)\right)^{n} x=\left(\int_{0}^{t} e^{\lambda s} d s-S(t)\right)^{n+1} z$, then

$$
(\lambda-A)^{n} x=(\lambda-A)^{n+1}\left[(\lambda-A)^{n-1} L_{n}(t) x+G_{n}(t) D_{\lambda}^{n+1}(t) z\right]
$$

Therefore $R(\lambda-A)^{n}=R(\lambda-A)^{n+1}$, hence $d(\lambda-A)<\infty$.

2. If $a\left(\int_{0}^{t} e^{\lambda s} d s-S(t)\right)<\infty$, there exists $n \in \mathbb{N}$ such that

$$
N\left(\int_{0}^{t} e^{\lambda s} d s-S(t)\right)^{n}=N\left(\int_{0}^{t} e^{\lambda s} d s-S(t)\right)^{n+1}
$$

Let $x \in N(\lambda-A)^{n+1}$ then $(\lambda-A)^{n+1} x=0$

$$
\begin{aligned}
(\lambda-A)^{n+1} x=0 & \Rightarrow\left(\int_{0}^{t} e^{\lambda s} d s-S(t)\right)^{n+1} x=0 \\
& \Rightarrow\left(\int_{0}^{t} e^{\lambda s} d s-S(t)\right)^{n} x=0 \\
(\lambda-A)^{n} x & =(\lambda-A)^{n} L_{n}(t)(\lambda-A)^{n} x
\end{aligned}
$$




$$
\begin{array}{ll} 
& +G_{n}(t)\left(\int_{0}^{t} e^{\lambda s} d s-S(t)\right)^{n} x \\
= & (\lambda-A)^{n-1} L_{n}(t)(\lambda-A)^{n+1} x \\
& +G_{n}(t)\left(\int_{0}^{t} e^{\lambda s} d s-S(t)\right)^{n} x \\
= & 0
\end{array}
$$

Therefore $N(\lambda-A)^{n}=N(\lambda-A)^{n+1}$, hence $a(\lambda-A)<\infty$.

3. If $d_{e}\left(\int_{0}^{t} e^{\lambda s} d s-S(t)\right)<\infty$, there exists $n \in \mathbb{N}$ such that

$$
\operatorname{dim} R\left(\int_{0}^{t} e^{\lambda s} d s-S(t)\right)^{n} / R\left(\int_{0}^{t} e^{\lambda s} d s-S(t)\right)^{n+1}<\infty
$$

Consider the application:

$$
\begin{aligned}
\psi: R(\lambda-A)^{n} & \rightarrow R\left(\int_{0}^{t} e^{\lambda s} d s-S(t)\right)^{n} / R\left(\int_{0}^{t} e^{\lambda s} d s-S(t)\right)^{n+1} \\
(\lambda-A)^{n} x & \mapsto\left(\int_{0}^{t} e^{\lambda s} d s-S(t)\right)^{n} x+R\left(\int_{0}^{t} e^{\lambda s} d s-S(t)\right)^{n+1}
\end{aligned}
$$

$\psi$ is well defined, linear, surjective. According to (1) we have

$$
N(\psi) \subseteq R(\lambda-A)^{n+1} \subseteq R(\lambda-A)^{n}
$$

According to the theorem of isomorphism we have $\operatorname{dim} R(\lambda-A)^{n} / N(\psi)=\operatorname{dim} R\left(\int_{0}^{t} e_{\infty}^{\lambda s} d s-S(t)\right)^{n} / R\left(\int_{0}^{t} e^{\lambda s} d s-S(t)\right)^{n+1}<$

Since

$$
R(\lambda-A)^{n+1} / N(\psi) \subseteq R(\lambda-A)^{n} / N(\psi)
$$

Then

$$
\operatorname{dim} R(\lambda-A)^{n+1} / N(\psi)<\infty
$$

According to the theorem of isomorphism we have

$$
\operatorname{dim} R(\lambda-A)^{n} / R(\lambda-A)^{n+1}<\infty
$$


Hence $d_{e}(\lambda-A)<\infty$.

4. If $a_{e}\left(\int_{0}^{t} e^{\lambda s} d s-S(t)\right)<\infty$, there exist $n \in \mathbb{N}$ such that

$$
\operatorname{dim} N\left(\int_{0}^{t} e^{\lambda s} d s-S(t)\right)^{n+1} / N\left(\int_{0}^{t} e^{\lambda s} d s-S(t)\right)^{n}<\infty
$$

We have $\operatorname{dim} N(\lambda-A)^{n+1} / N(\lambda-A)^{n}<\infty$.

Indeed consider the application:

$$
\begin{aligned}
\varphi: N(\lambda-A)^{n+1} & \rightarrow N\left(\int_{0}^{t} e^{\lambda s} d s-S(t)\right)^{n+1} / N\left(\int_{0}^{t} e^{\lambda s} d s-S(t)\right)^{n} \\
x & \mapsto x+N\left(\int_{0}^{t} e^{\lambda s} d s-S(t)\right)^{n}
\end{aligned}
$$

$\varphi$ is well defined, linear, and $N(\varphi) \subseteq N(\lambda-A)^{n} \subseteq N(\lambda-A)^{n+1}$.

According to the theorem of isomorphism we have

$$
\begin{aligned}
\operatorname{dim} N(\lambda-A)^{n+1} / N(\varphi) & =\operatorname{dim} \operatorname{Im}(\varphi) \\
& \leq \operatorname{dim} N\left(\int_{0}^{t} e^{\lambda s} d s-S(t)\right)^{n+1} / N\left(\int_{0}^{t} e^{\lambda s} d s-S(t)\right)^{n} \\
& <\infty
\end{aligned}
$$

Then $\operatorname{dim} N(\lambda-A)^{n} / N(\varphi)<\infty$. According to the theorem of isomorphism we have $\operatorname{dim} N(\lambda-A)^{n+1} / N(\lambda-A)^{n}<\infty$ hence $a_{e}(\lambda-A)<\infty$.

5. If $\int_{0}^{t} e^{\lambda s} d s-S(t)$ is Drazin invertible, then $d\left(\int_{0}^{t} e^{\lambda s} d s-S(t)\right)<\infty$ and $a\left(\int_{0}^{t} e^{\lambda s} d s-S(t)\right)<\infty$ then $\left.d(\lambda-A)\right)<\infty$ and $a(\lambda-A)<\infty$, hence $\lambda-A$ is invertible Drazin.

Corollary 3. Let $S(t)_{t \geq 0}$ be an integrated semigroup on $X$ with generator $A$. Then for all $t>0$ :

$$
\begin{gathered}
\int_{0}^{t} e^{s \sigma_{d e s c}(A)} d s \subseteq \\
\int_{0}^{t} e^{s \sigma_{d e s c}^{e}(A)} d s \subseteq \sigma_{d e s c}^{e}(S(t)), \int_{0}^{t} e^{s \sigma_{a s c}(A)} d s \subseteq \sigma_{a s c}(S(t)), \int_{0}^{t} e^{s \sigma_{a s c}^{e}(A)} s \subseteq \sigma_{a s c}^{e}(S(t)) \\
\int_{0}^{t} e^{s \sigma_{D}(A)} d s \subseteq \sigma_{D}(S(t))
\end{gathered}
$$

Theorem 5. Let $(S(t))_{t \geq 0}$ an integrated semigroup on $X$ with generator $A$. Then for all $t>0$ we have 
1. If $\int_{0}^{t} e^{\lambda s} d s-S(t)$ is a upper semi-Browder operator, then $\lambda-A$ is a upper semi-Browder operator.

2. If $\int_{0}^{t} e^{\lambda s} d s-S(t)$ is a lower semi-Browder operator, then $\lambda-A$ is a lower semi-Browder operator.

3. If $\int_{0}^{t} e^{\lambda s} d s-S(t)$ is a Browder operator, then $\lambda-A$ is a Browder operator.

Proof. 1. If $\int_{0}^{t} e^{\lambda s} d s-S(t)$ is a upper semi-Browder operator, then $\int_{0}^{t} e^{\lambda s} d s-S(t)$ is a upper semi-Fredholm and $d\left(\int_{0}^{t} e^{\lambda s} d s-S(t)\right)<\infty$. According to theorem3 and theorem4 we have $\lambda-A$ is a upper semiFredholm and $d(\lambda-A)<\infty$, hence $\lambda-A$ is a upper semi-Browder operator.

2. If $\int_{0}^{t} e^{\lambda s} d s-S(t)$ is a lower semi-Browder operator, then $\int_{0}^{t} e^{\lambda s} d s-S(t)$ is a lower semi-Fredholm and $a\left(\int_{0}^{t} e^{\lambda s} d s-S(t)\right)<\infty$. According to theorem3 and theorem4 we have $\lambda-A$ is a lower semi-Fredholm and $d(\lambda-A)<\infty$, hence $\lambda-A$ is a lower semi-Browder operator.

3. obvious.

Corollary 4. Let $S(t)_{t \geq 0}$ be an integrated semigroup on $X$ with generator $A$. Then for all $t>0$ :

$$
\begin{aligned}
\int_{0}^{t} e^{s \sigma_{\mathcal{B}_{+}}(A)} s \subseteq & \sigma_{\mathcal{B}_{+}}(S(t)), \int_{0}^{t} e^{s \sigma_{\mathcal{B}_{-}}(A)} s \subseteq \sigma_{\mathcal{B}_{-}}(S(t)) \\
& \int_{0}^{t} e^{s \sigma_{\mathcal{B}}(A)} s \subseteq \sigma_{\mathcal{B}}(S(t))
\end{aligned}
$$

\section{References}

[1] W. Arendt, Vector-valued Laplace transforms and Cauchy problems, Israel J. Math., 59 (1987), 327-352.

[2] K.J. Engel and R. Nagel, One-Parameter Semigroups for Linear Evolution Equations, Graduate Texts in Mathematics, Volume 194, Springer-Verlag, New York, 2000.

[3] D.C. Lay, Spectral analysis using ascent, descent, nullity and defect, Math. Ann., 184 (1970), 197-214.

[4] V. Müller, Spectral Theory of Linear Operators and Spectral Systems in Banach Algebras, 2-nd Edition, Oper. Theory Advances and Applications, Volume 139 (2007).

[5] A. Pazy, Semigroups of Linear Operators and Applications to Partial Differential Equations, Applied Mathematical Sciences, Volume 44, Springer-Verlag, New York 1983. 